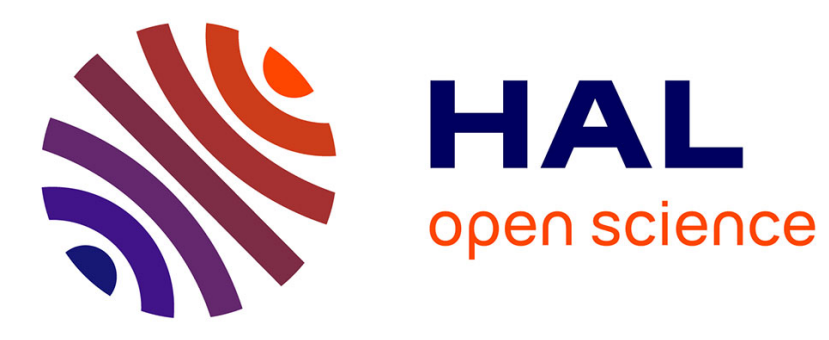

\title{
Unified explanation for rogue waves in optical fibers
} Fabio Biancalana, Andrea Armaroli, Claudio Conti

\section{To cite this version:}

Fabio Biancalana, Andrea Armaroli, Claudio Conti. Unified explanation for rogue waves in optical fibers. SPIE Newsroom, 2015, Optoelectronics \& Communications, 10.1117/2.1201507.006035 . hal01193137

\section{HAL Id: hal-01193137 \\ https://hal.science/hal-01193137}

Submitted on 4 Sep 2015

HAL is a multi-disciplinary open access archive for the deposit and dissemination of scientific research documents, whether they are published or not. The documents may come from teaching and research institutions in France or abroad, or from public or private research centers.
L'archive ouverte pluridisciplinaire HAL, est destinée au dépôt et à la diffusion de documents scientifiques de niveau recherche, publiés ou non, émanant des établissements d'enseignement et de recherche français ou étrangers, des laboratoires publics ou privés. 


\section{Unified explanation for rogue waves in optical fibers}

Fabio Biancalana, Andrea Armaroli, and Claudio Conti

The concept of an 'energy landscape' is used to model the formation of rogue solitons.

So-called rogue waves (also known as 'freak' or 'killer' waves) are waves with extremely large amplitudes. They appear from nothing and then suddenly disappear. The rogue wave concept has it origins in the field of oceanography, but it has also been studied in many different branches of physics (e.g., optical guided waves and plasma waves), and in complex dynamic systems (e.g., social sciences and financial markets). ${ }^{1}$ The nature and origin of rogue waves, however, are not fully understood.

Large nonlinearities within photonic crystal fibers (i.e., a form of light-matter interaction) cause intense incoming light pulses to break up into trains of short pulses, which are known as solitons. These solitons exhibit particle-like behavior, and their shape tends to be preserved during propagation. When the solitons are sufficiently short (typically less than 1ps), a cascade of high-order linear and nonlinear phenomena results in the generation of a broad optical spectrum, which is known as a supercontinuum. ${ }^{2}$ During this process, rare events can occur in unpredictable manners. For instance, an unusually high intensity soliton can appear at the fiber output. ${ }^{3}$ Such an event is considered as a fiber-optical analogue of oceanic rogue waves. Several numerical and experimental studies have been conducted to characterize and control optical rogue waves ${ }^{4}$ and rogue solitons. The main theoretical explanation derived from these investigations is that these phenomena correspond to exact solutions of universal mathematical models, e.g., the nonlinear Schrödinger (NLS) equation. ${ }^{5}$ The solutions of these models are of fundamental theoretical interest and have applications in many contexts. However, they cannot be used to explain the statistical properties of rogue solitons in optical fibers, nor the observed variety of peak amplitudes.

We developed a new model that can explain the properties of rogue solitons in optical fibers. We used statistical physics methods, borrowed from the field of spin glasses, ${ }^{6}$ to model the weak interactions between fiber solitons. We were thus able

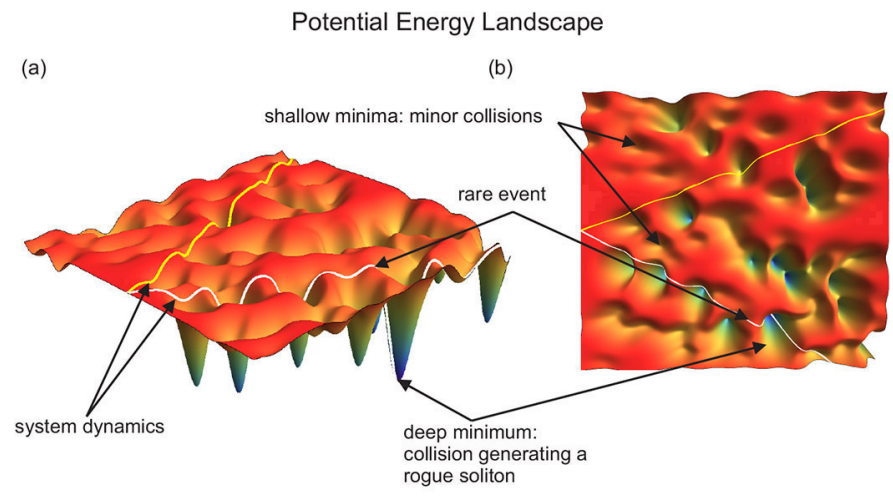

Equilibria of the weak-interaction model

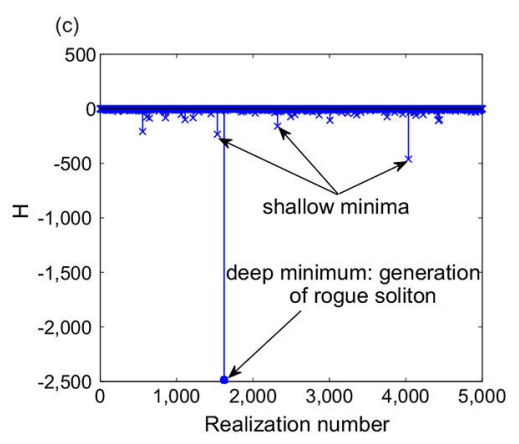

Figure 1. (a) and (b) Illustration of the potential energy landscape. The trajectories of two different potential energy levels are shown. (c) Construction of the equivalent energy landscape for the weak interactions of nonlinear Schrödinger solitons (in terms of a list of numerically computed values). H: Depth of energy minima.

to build a model of an 'energy landscape,' which is generated by the weak interaction between adjacent solitons. ${ }^{7}$ We took this concept of an energy landscape from the physics of complex systems (e.g., glass phase transitions, protein folding, and chemical reactions). ${ }^{8}$ The interaction of many degrees of freedom gives rise to the complex behavior, i.e., the system is intrinsically unpredictable. Our energy landscape consists of many

Continued on next page 
(a1)

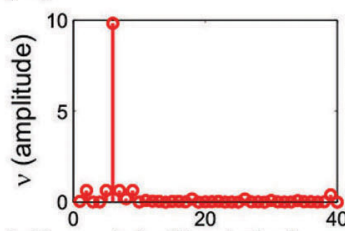

(a3) $\mathrm{k}$ (soliton index)

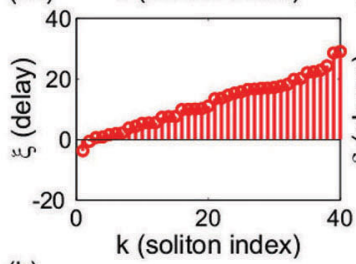

(b)

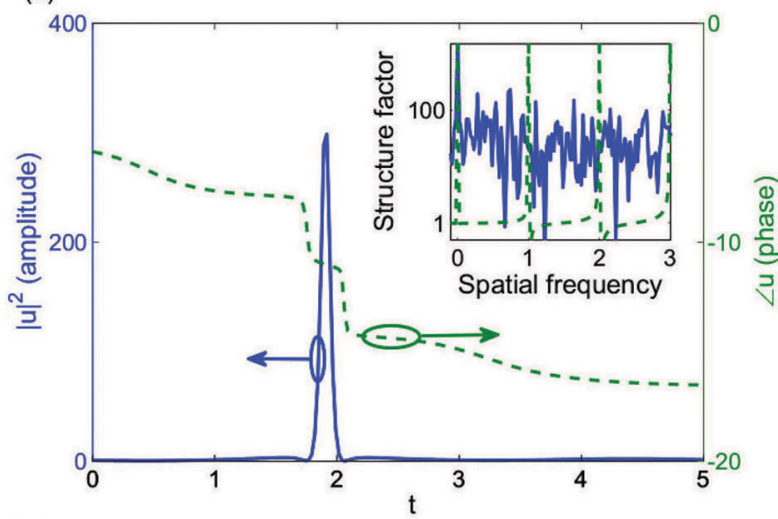

(c)

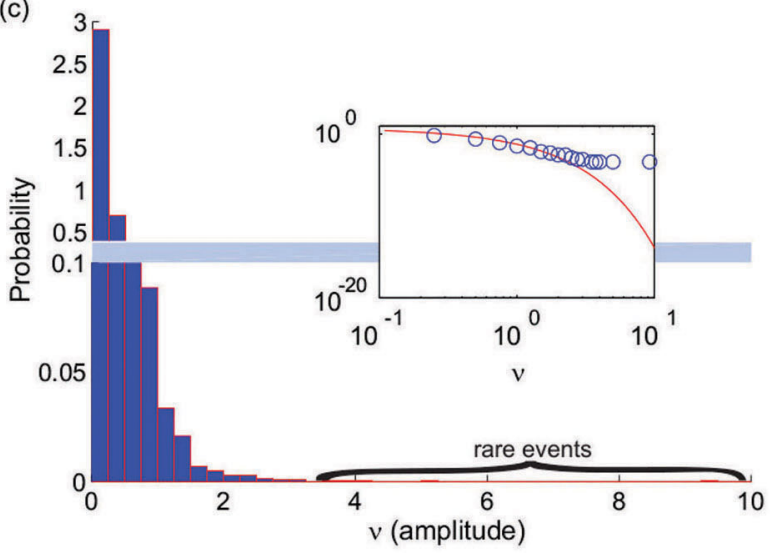

Figure 2. Characteristics of the equilibria of the weak interaction soliton model. The solution that exhibits an exceptionally large peak is shown in (a1) and in (a2), where it is surrounded by smaller peaks with mismatched velocity. The corresponding temporal profiles of intensity and phase are shown in (b). The statistical distribution of the numerically computed amplitudes of the equilibria is shown in (c). The inset shows the data fit to a Weibull distribution (on a logarithmic scale). t: Time. u: Electric field amplitude.
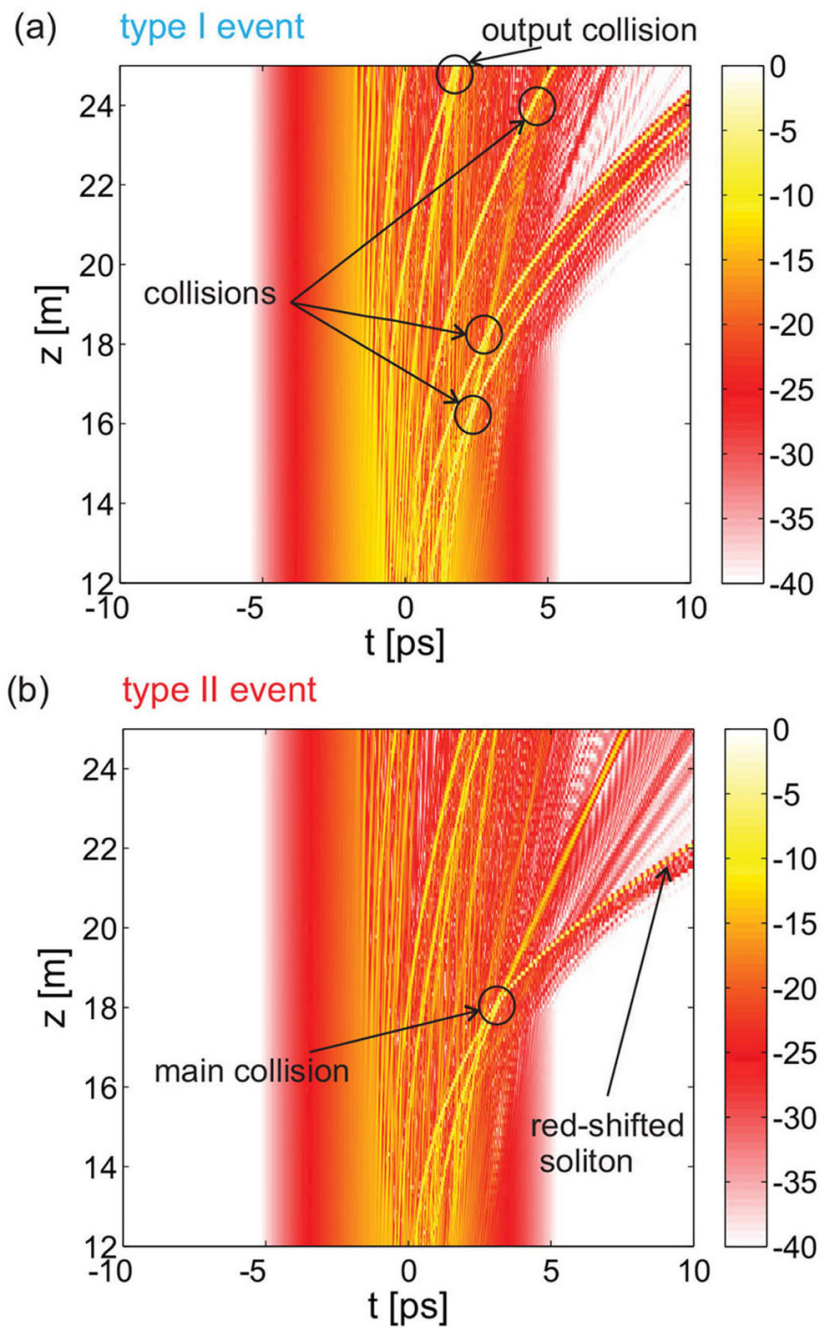

Figure 3. Light propagation simulations reveal two different dynamical processes that give rise to extreme amplitude peaks at a fiber output. (a) During a type I event, a collision occurs at the fiber output after a sequence of minor collisions. (b) In a type II event, a major early collision transfers a large amount of energy to a strong soliton, which is then red-shifted. The most important collision events are highlighted with circles. $z$ : Propagation distance along the optical fiber.

minima with different depths, as illustrated in Figure 1(a) and Figure 1(b). Different initial conditions led to completely different evolutions of our system, i.e., the energy is free to hop between different minima and explore a variety of permitted configurations. We used our model to identify the equilibria of weak soliton interactions. We found that the equilibria 


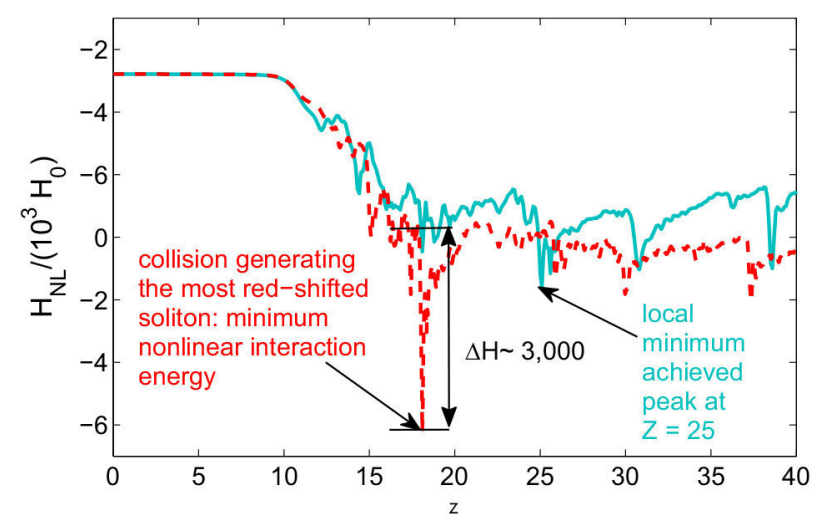

Figure 4. Evolution of the potential energy of the simulated system. Blue and red lines correspond to type I and II events illustrated in Figure 3. $H_{N L}$ : Nonlinear height. $H_{0}$ : Initial height. $z$ : Propagation distance along the optical fiber.

corresponded to the collisions that originally generated the rogue solitons in optical fibers.

We applied our approach to the universal NLS equation, which describes the propagation of light in an optical fiber within the anomalous dispersion regime. ${ }^{9}$ We were able to simply derive the necessary system of ordinary differential equations ${ }^{7}$ with the use of a variational ansatz and an equation that relates the amplitude, speed, temporal delay, and phase of each soliton. We used a numerical root finding technique-for a large soliton number $(N)$-to calculate the equilibria of the resulting system. We repeated this same procedure for many different initial random guesses, and we were thus able to find a large number of equilibria. The resultant family of solutions represents the energy landscape of the soliton interaction.

We also examined the statistical distribution of the amplitudes in our model. We searched for exceptional solutions that exhibited extreme amplitudes. Among these solutions, we found an intense pulse that exhibited a very large peak and was surrounded by smaller oscillations, as illustrated in Figure 2(a) and Figure 2(b). Although this solution appears to be similar to the well-known Akhmediev breather (nonlinear wave) and Peregrine soliton, ${ }^{5}$ this perception is misleading because we also found solutions with multiple peaks. In addition, the amplitude ratio of the main soliton to its neighbors does not coincide with the prediction for these exact solutions. Interestingly, we found that high-intensity isolated peaks corresponded to minima of the integrated Hamiltonian density-see Figure 1(c)—which is equivalent to the total energy of the system. ${ }^{10}$ Moreover, the statistical distribution of the amplitudes is a Weibull distribution, 4 which exactly matches our experimental observations: see
Figure 2(b). Furthermore, the moments of this distribution are in the same range as those that are measured for rogue solitons in optical fibers. ${ }^{11}$

In addition, we simulated light propagation in a realistic photonic crystal fiber. ${ }^{9}$ As expected, ${ }^{12}$ we observed the generation of rogue events as collisions between solitons in the proximity of the fiber output - see Figure 3(a) —or as large amplitude solitons at the red edge of the spectrum-see Figure 3(b) - because of Raman self-frequency shift. We found the collision events that occurred during supercontinuum generation (SCG) matched the temporal profile of the energy landscape equilibria: see Figure 2(b). The red-shifted pulses originated from extreme early collisions, whereas the SCG collisions resulted from a sequence of many minor collisions. Each collision corresponds to a minimum

in the interaction energy between solitons, which we calculated by integrating the nonlinear terms of the Hamiltonian density (see Figure 4). ${ }^{10}$ This provides the strongest indication that collisions inside the pulse train are the equilibria of the energy landscape and are generated by the interactions of adjacent solitons.

We have proposed an explanation for the appearance of extreme peaks at the output of an optical fiber, i.e., rogue solitons. Our approach is based on a simplified interaction model for the behavior of neighboring solitons. ${ }^{6}$ Our method allows us to build an energy landscape. The equilibria of this landscape correspond to the collisions between the light pulses that are generated during the initial propagation stages. We have mathematically proven that the interaction energy represents local minima at those points and that the peak statistical distribution corresponds to the amplitude distribution of equilibria. The generality and universality of our concept makes it particularly useful to scientific and engineering communities involved in the study and control of freak waves. In our future studies, we will investigate if the energy landscape can be used to explain other types of rogue waves (e.g., freak waves that are generated by modulational instabilities in optical fibers or in water) and the rogue waves that are observed in the chaotic regime of microring resonators. We eventually hope to obtain a unified understanding of all natural rogue wave phenomena.

Fabio Biancalana and Andrea Armaroli acknowledge the support of the German Max Planck Society for the Advancement of Science for this work. 


\section{Author Information}

\section{Fabio Biancalana}

Heriot-Watt University

Edinburgh, United Kingdom

Fabio Biancalana has been an associate professor since 2012. He also leads the nonlinear photonic nanostructures research group at the Max Planck Institute for the Science of Light, Germany.

\section{Andrea Armaroli}

Advanced National College of Applied Science and Technology (ENSSAT)

University of Rennes

Lannion, France

Andrea Armaroli obtained his $\mathrm{PhD}$ from the University of Ferrara, Italy, and the Grenoble Institute of Technology, France. He then completed a two-year postdoctoral fellowship at the Max Planck Institute for the Science of Light, Germany. He is now a research assistant at the Optical Function for Information Technology and ENSSAT in France.

Claudio Conti

Institute for Complex Systems (ISC)

Italian National Research Council (CNR)

Rome, Italy

Claudio Conti obtained his PhD in 2002 and is now the director of the ISC-CNR. Formerly, he was an associate professor at the Sapienza University of Rome, Italy, and of the CNR at the ISC. He was awarded a New Talent Grant from the Enrico Fermi Center and a Starting Independent Research Grant from the European Research Council in 2008. In 2010, he was awarded a Humboldt Research Fellowship for experienced researchers at the Max Planck Institute for the Science of Light, Germany.

\section{References}

1. M. Onorato, S. Residori, U. Bortolozzo, A. Montina, and F. T. Arecchi, Rogue waves and their generating mechanisms in different physical contexts, Phys. Rep. 528, pp. 47-89, 2013.

2. J. M. Dudley, G. Genty, and S. Coen, Supercontinuum generation in photonic crystal fiber, Rev. Mod. Phys. 78, pp. 1135-1184, 2006.

3. D. R. Solli, C. Ropers, P. Koonath, and B. Jalali, Optical rogue waves, Nature $\mathbf{4 5 0}$ pp. $1054-1057,2007$

4. J. M. Dudley, G. Genty, and B. J. Eggleton, Harnessing and control of optical rogue waves in supercontinuum generation, Opt. Express 16, pp. 3644-3651, 2008.

5. J. M. Dudley, G. Genty, F. Dias, B. Kibler, and N. Akhmediev, Modulation instability, Akhmediev breathers and continuous wave supercontinuum generation, Opt. Express 17, pp. 21497-21508, 2009.

6. A. Armaroli, C. Conti, and F. Biancalana, Rogue solitons in optical fibers: a dynamical process in a complex energy landscape?, Optica 2, pp. 497-504, 2015.

7. I. M. Uzunov, V. S. Gerdjikov, M. Gölles, and F. Lederer, On the description of $N$-soliton interaction in optical fibers, Opt. Commun. 125, pp. 237-242, 1996.

8. P. G. Debenedetti and F. H. Stillinger, Supercooled liquids and the glass transition Nature 410, pp. 259-267, 2001.

9. G. Agrawal, Nonlinear Fiber Optics, 5th ed., p. 648, Academic Press, 2012.

10. C. Sulem and P.-L. Sulem, Nonlinear Schrodinger Equation: Self-Focusing Instability and Wave Collapse, p. 355, Springer-Verlag Berlin and Heidelberg GmbH \& Co. K, 1999.

11. S. T. Sørensen, O. Bang, B. Wetzel, and J. M. Dudley, Describing supercontinuum noise and rogue wave statistics using higher-order moments, Opt. Commun. 285 pp. 2451-2455, 2012.

12. G. Genty, C. M. de Sterke, O. Bang, F. Dias, N. Akhmediev, and J. M. Dudley, Collisions and turbulence in optical rogue wave formation, Phys. Lett. A 374, pp. 989996, 2010 